- Cambridge medical students expressed feelings of loneliness and isolation during medical student placements, where they could be allocated in small groups (often 2 or 3 individuals) to remote areas of Cambridgeshire.

- Care homes residents reported a feeling of lack of social integration with those outside of their care homes.

Aims Our aim was to create an intervention that:

1. Tackled loneliness in students and elderly residents in Cambridge.

2. Established social integration across different generations.

Intervention Following a stakeholder engagement and workshop with: medical student representatives, Cambridge university executive board and Cambridge local council care home lead; a pilot pen-pal scheme was launched between care home residents and Cambridge medical students.

Measurement of impact and improvement

Impact will be measured following a year of letter-writing to assess:

1. A change in score of feeling of loneliness.

2. Understanding another generation.

Provisional feedback has been positive, with all 10 pairs in the pilot recommending the scheme to a friend or family, and reporting that they have had interesting and enjoyable conversations'.

Conclusion Pen-to-paper is a unique and engaging way to tackle the complex problem of social isolation and loneliness in two at high-risk groups. The intervention has been well received by both groups and we are extending the intervention to residents in warden controlled/sheltered accommodation.

\section{QR CODE, DOCUMENT STUDIO AND PATIENT SAFETY}

Mohammad Irfan, Najla Al Mutairi, Muna Bhutta. Al-Iman General Hospital, Riyadh, Saudi Arabia

\subsection{6/leader-2019-FMLM.71}

\section{Aims}

1. Think Out Of Box

2. To know about application of technology(google add on) in Incident Reporting to overcome barriers, such as, Complex system of reporting, time consuming, manpower and supplies issues, feedback issues, fear of punitive action, and inability to report as anonymous.

Methods Google add ons like spredsheet, document studio and QR code was designed and used for incident reporting, getting responses and giving feedbacks.

Results Incident reporting was increased to nearly 3 to 4 times. Incident reporting barriers were removed. Monitoring of the compliance to the essential safety standards(Blood transfusion reaction, Patient identification, Safe and correct procedure, venous thromboembolism prophylaxis) was made easy. Even Patients started to contribute in Incident reporting.

Conclusion With the help of the applied google tools, we were able to have a direct live access to the dashboard for Incident reporting and also helped to monitor Indicators of patient safety, particularly, the timeliness of the OVR(Occurance Variance Report) System.

\section{REDUCTION OF NEEDLESTICK INJURIES AMONG NURSES AND HEALTHCARE ASSISTANTS THROUGH AN INTERVENTION: NATIONAL HOSPITAL SRI LANKA (NHSL)}

${ }^{1}$ Imesh Dilshara Prathapasinghe, ${ }^{2}$ Samath D Dharmarathne. ${ }^{1}$ Post Doctor of Medicine Trainee in Medical Administration, Sherwood Forest Hospitals, NHS Foundation Trust, UKi ${ }^{2}$ Professor of Community Medicine, Faculty of Medicine, University of Peradeniya, Sri Lanka

\subsection{6/leader-2019-FMLM.72}

Introduction Cutaneous injuries, resulting from needle sticks, injection devices and sharps are a major issue for all health care workers and cause a considerable threat of spreading blood-related infections like HIV.

Aim To reduce NSI among nurses (NO) and health care assistants (HCA) in the NHSL, by assessing the current gaps in the Knowledge, attitude and practice and designing intervention to mitigate the harm and reduce the injuries.

Method An Interventional study was conducted in three components, pre-interventional, interventional and post interventional. Random sampling technique was applied to select the appropriate number of nurses and health care assistants.

Pre-interventional component:

To identify the gaps in the present managerial practices on NSI, a pre-tested structured questionnaire on knowledge, attitude and practices was administered.

Interventional component:

Two separate in-service programmes were conducted for both categories. WHO recommended injection safety tool kit was also introduced.

Post-interventional component:

Outcome of the interventions were assessed by measuring the pre- and post-test knowledge, attitude, and practice of the same participants. The same tool was administered.

Results

1. Both Groups (NO and HCA) showed a highly significance different after interventions, on Reporting system for NSI: $p$ value $(0.05,0.001)$. Awareness on Post Exposure prophylaxis (0.003, 0.049).

2. Non-significance difference among nursing officers on Knowledge attitude and practice. All the $\mathrm{p}$ values observed $0.05<$ and $\mathrm{z}$ evident with negative findings.

3. Only Knowledge component among the HCA indicates a significant difference.

Conclusion and recommendation It was recommended to conduct more awareness programs and training modules on post exposure management of NSI because it has shown positive Results in both categories.

WHO injection safety tool kit has also shown positive Results.

\section{TEA BREAK TALK: A WELLBEING INITIATIVE FOR JUNIOR DOCTORS}

Btari Adityani, Cristina Musat*. University Hospitals Plymouth NHS Trust, UK

\subsection{6/leader-2019-FMLM.73}

Background The 2019 BMA report on 'Caring for the mental health of the medical workforce' found that $80 \%$ of doctors are at high risk of burnout with junior doctors being most at risk. The 2018 GMC Training Environment Report also found that $25 \%$ of doctors in training felt burnout associated with 